\title{
A pooling-based genome-wide analysis identifies new potential candidate genes for atopy in the European Community Respiratory Health Survey (ECRHS) \\ Francesc Castro-Giner ${ }^{\dagger 1,2,3}$, Mariona Bustamante ${ }^{\dagger 3,4}$, Juan Ramon González ${ }^{1,2,3}$, Manolis Kogevinas ${ }^{1,2,3,5}$, Deborah Jarvis ${ }^{6}$, Joachim Heinrich7, Josep-Maria Antó1,2,3,8, Matthias Wjst ${ }^{9}$, Xavier Estivill ${ }^{3,4,8}$ and Rafael de $\mathrm{Cid}^{* 3,4,10}$
}

Address: ${ }^{1}$ Centre for Research in Environmental Epidemiology (CREAL), Barcelona, Spain, ${ }^{2}$ Municipal Institute of Medical Research (IMIMHospital del Mar), Barcelona, Spain, ${ }^{3}$ Public Health and Epidemiology Network Biomedical Research Center (CIBERESP), Barcelona, Spain, ${ }^{4}$ Genes and Disease Program, Center for Genomic Regulation (CRG), Barcelona, Spain, ${ }^{5}$ Medical School, University of Crete, Heraklion, Greece, ${ }^{6}$ Respiratory Epidemiology and Public Health Group, National Heart and Lung Institute, Imperial College, London, UK, ${ }^{7}$ Institute of Epidemiology, Helmholtz Zentrum München, Munich, Germany, ${ }^{8}$ Department of Health and Experimental Sciences, University Pompeu Fabra, Barcelona, Spain, ${ }^{9}$ German Research Center for Environmental Health, Helmholtz Centre GSF, Munich, Germany and ${ }^{10}$ CEA, Institute de Genomique. Centre National de Genotypage (CNG), Evry, France

Email: Francesc Castro-Giner - fcastro@creal.cat; Mariona Bustamante - mariona.bustamante@crg.es; Juan Ramon González - jrgonzalez@creal.cat; Manolis Kogevinas - kogevinas@creal.cat; Deborah Jarvis - d.jarvis@imperial.ac.uk; Joachim Heinrich - joachim.heinrich@helmholtz-muenchen.de; Josep-Maria Antó - jmanto@creal.cat; Matthias Wjst - m@wjst.de; Xavier Estivill - xavier.estivill@crg.es; Rafael de Cid* - decid@cng.fr

* Corresponding author †Equal contributors

Published: 6 December 2009

BMC Medical Genetics 2009, 10:128 doi:10.1186/147/-2350-10-128

This article is available from: http://www.biomedcentral.com/ I 47/ -2350/10/128

(c) 2009 Castro-Giner et al; licensee BioMed Central Ltd.

This is an Open Access article distributed under the terms of the Creative Commons Attribution License (http://creativecommons.org/licenses/by/2.0), which permits unrestricted use, distribution, and reproduction in any medium, provided the original work is properly cited.
Received: I April 2009

Accepted: 6 December 2009

\begin{abstract}
Background: Asthma and atopy are complex phenotypes with shared genetic component. In this study we attempt to identify genes related to these traits performing a two-stage DNA pooling genome-wide analysis in order to reduce costs. First, we assessed all markers in a subset of subjects using DNA pooling, and in a second stage we evaluated the most promising markers at an individual level.

Methods: For the genome-wide analysis, we constructed DNA pools from 75 subjects with atopy and asthma, 75 subjects with atopy and without asthma and 75 control subjects without atopy or asthma. In a second stage, the most promising regions surrounding significant markers after correction for false discovery rate were replicated with individual genotyping of samples included in the pools and an additional set of 429 atopic subjects and 222 controls from the same study centres.

Results: Homo sapiens protein kinase-like protein SgK493 (SGK493) was found to be associated with atopy. To lesser extent mitogen-activated protein kinase 5 (MAP3K5), collagen type XVIII alpha I (COLI8AI) and collagen type XXIX alpha I (COL29AI) were also found to be associated with atopy. Functional evidences points out a role for MAP3K5, COLI 8AI and COL29A I but the function of SGK493 is unknown.
\end{abstract}

Conclusion: In this analysis we have identified new candidate regions related to atopy and suggest SGK493 as an atopy locus, although these results need further replication. 


\section{Background}

Asthma and atopy are complex phenotypes with environmental and genetic determinants. Several chromosomal regions and candidate genes have been implicated in asthma or atopy susceptibility $[1,2]$. Genome wide association (GWA) studies have been successful in the identification of loci contributing to complex diseases, included asthma [3-5]. However a limitation of GWA studies is the high economic cost required. A cost-effective alternative is the use of pooled DNA followed by individual genotyping [6-8]. In this study, we performed a pooling-based GWA for asthma and atopy, with further validation of most promising regions in the individual pooled samples as well as in a second set of cases and controls.

\section{Methods \\ Pooling GWA}

In the first stage we conducted a GWA analysis using a DNA-pooling approach. Genotyping was performed using the Illumina HumanHap 300 Whole-Genome Genotyping BeadChip. The genome-wide analysis was conducted in three different sets of pooled samples: (1) subjects with atopy and asthma; (2) subjects with atopy and without asthma, and (3) control subjects with neither asthma nor atopy (Table 1). Samples for this analysis were randomly selected from United Kingdom, Spain and Germany cohorts of the European Community Respiratory Health Survey (ECRHS) study [9]. Atopy was defined as sensitization (IgE levels $>0.35 \mathrm{kU} / \mathrm{L}$ ) to specific allergens
(D. Pteronyssinus, cat, timothy grass. or C. herbarum). Asthma was defined as the presence of attacks of asthma in the last 12 months or taking currently medication for asthma. All asthmatics subjects had also atopy. For pool construction, DNA samples were diluted and measured twice by double stranded DNA quantification using PicoGreen ${ }^{\oplus}$ dsDNA reagent Kit (Invitrogen), and then normalized at $50 \mathrm{ng} / \mu \mathrm{l}$. After visual inspection in $0.7 \%$ alkaline agarose gels, selected DNA samples were pooled in three independent pools of 75 individuals each one. We have controlled the quality of the Illumina genotyping process including a sample from HapMap (GM12873) with known genotype for the panel of $300 \mathrm{~K}$ markers. We verified the concordance of genotypes with the HapMap database (concordance $=99.91 \%$ ) and inconsistent single nucleotide polymorphisms (SNPs) were excluded from the analysis $(n=296,0.09 \%)$. A pool of HapMap $(n=57)$ subjects were also performed and genotyped to validate the allele frequency estimation. SNPs with a poor performance in the estimation of allele frequency were excluded from the analysis ( $\mathrm{n}=3271,1.03 \%)$ using a threshold of $>12 \%$ of difference, as shown in previous reports $[7,10]$, Finally, three replicates of each pool of ECRHS subjects were constructed to account for sampling errors in the analysis.

Allele frequencies for each SNP were estimated from pooled samples correcting by the ratio of intensity of both alleles [11]. Allele frequencies in each pool were com-

Table I: Population characteristics of asthma and atopy analyzed samples

\begin{tabular}{|c|c|c|c|c|c|c|}
\hline & \multicolumn{3}{|c|}{ Pooling Samples } & \multicolumn{3}{|c|}{ Replication samples } \\
\hline & Control & Atopy & Atopic asthma & Control & Atopy & Atopic asthma subsample \\
\hline Subjects, $n$ & 75 & 75 & 75 & 222 & 429 & 198 \\
\hline Subjects excluded*, n (\%) & $14(19)$ & $17(23)$ & $14(19)$ & $47(2 \mathrm{I})$ & $98(23)$ & $48(24)$ \\
\hline Age, mean (sd) & $35.87(6.79)$ & $32.21(7.78)$ & $32.07(6.95)$ & $35.36(7.16)$ & $33.32(7.15)$ & $33.19(7.14)$ \\
\hline \multicolumn{7}{|l|}{ Gender } \\
\hline Males, n (\%) & $37(49)$ & $38(5 \mathrm{I})$ & $37(49)$ & $106(48)$ & $205(48)$ & $84(42)$ \\
\hline Females, n (\%) & $38(5 \mathrm{I})$ & $37(49)$ & $38(5 \mathrm{I})$ & $116(52)$ & $224(52)$ & $114(58)$ \\
\hline \multicolumn{7}{|l|}{ Smoking } \\
\hline Current, n (\%) & $19(25)$ & $23(31)$ & $28(38)$ & $56(25)$ & $100(23)$ & $37(19)$ \\
\hline Ex, n (\%) & $20(27)$ & $18(24)$ & $16(22)$ & $58(26)$ & $96(22)$ & $47(24)$ \\
\hline Never, n (\%) & $36(48)$ & $34(45)$ & $30(4 I)$ & $107(48)$ & $232(54)$ & II4 (58) \\
\hline
\end{tabular}

*Subjects excluded at the individual genotyping (stage 2) 
pared using the $1 \mathrm{df}$ chi-square T statistic for testing differences between two proportions accounting for experimental and sampling errors [11]. Multiple testing was controlled using a false discovery rate (FDR) at 5\% [12]. Allele frequency estimation was validated using a pool of 57 samples from HapMap whose individual genotypes for $300 \mathrm{~K}$ Illumina panel were previously known. Predicted and real allele frequencies showed a strong correlation (Pearson correlation coefficient, $r=0.99$ ).

\section{Individual genotyping in ECHRS}

In a second stage, replication was performed by individual genotyping of subjects included in the pools $(n=225)$ and in an additional set of 429 atopic subjects (46\% with asthma) and 222 controls subjects randomly selected from the ECRHS study. Only promising SNPs that were still significant after correction by multiple testing were followed up. Target regions were delimited by non significant SNPs ( $p>0.05)$ upstream and downstream from the SNP significant associated after multiple testing corrections in the first stage (see Additional file 1: table S1). Complex regions with genomic structural variants were discarded for this study (chr 1 and chr 5) [13]. For the saturation we used a tag SNP approach forcing the inclusion of the polymorphisms detected in the first stage, or their perfect tags $\left(r^{2} \geq 0.8\right)$. From the HapMap project data set, we utilized genotypes from the public release 21a (phase II; NCBI35, dbSNPb125) corresponding to 90 individuals from the CEPH 30 trios of European descent. The selection of tag SNPs was performed using the pair-tagging strategy implemented in Haploview software (v.4.0) with a MAF $\geq 0.05$ and an $\mathrm{r}^{2} \geq 0.8$ [14]. In addition to HapMap SNPs, four other polymorphisms previously described to be associated with atopic dermatitis and situated in one of the targeted regions (COL29A1) were included [15]. The 53 SNPs selected were genotyped in individual samples using SNPlex technology (Applied Biosystems).

Genotyping quality was controlled by including negative controls and internal positive controls consisting in four replicates of two HapMap reference samples. Both, genotype concordances with the HapMap database and among replicates, were verified. Genotype data caring was done using SNP analysis to results software (SNPator) http:// www.snpator.com/. Forty-five out of 225 pooled samples and 145 out of 651 replication samples were dropped from the individual analysis due to low volume of DNA available or low concentration (Table 1). Seven out of 53 genotyped SNPs were excluded from the analysis since deviation from Hardy-Weinberg equilibrium (HWE) (in controls $\mathrm{p}<0.05$ ) or showed a genotyping rate under $80 \%$. The final replication set included 46 SNPs with an overall genotyping rate of $97 \%$ in 686 samples (Table 1). Genotyping assays were performed at the Barcelona Node of the "Centro Nacional de Genotipado" (CeGen) in Spain.
The statistical analysis for data derived from individual genotyping was performed assuming an additive genetic model and using logistic regression using R statistical software package [16]. Models were adjusted for age, sex and smoking status. Country of origin was considered in the regression models in order to discard confounding by population stratification [17]. Linkage disequilibrium (LD) blocks were estimated according to Gabriel et al. [18] method implemented in Haploview.

\section{SGK493 tissue expression}

A human RNA tissue panel (Stratagene) including brain, heart, kidney, liver, lung, placenta, skeletal muscle, spleen, testis, ovary and thymus was used to evaluate the expression of SGK493. One $\mu \mathrm{g}$ of RNA was converted to cDNA using the SuperScript III First-Strand Synthesis System for RT-PCR (Invitrogen). SGK493 and the GAPDH genes were amplified in independent PCRs using the following primers SGK493-F: 5'-AAGTGACGGACCTGGATGAC-3' and SGK493-R: 5'-TGTGAGGCAG GAGG TAT GT G-3' and GAPDH-F: 5'-ACCCAGAAGACTGTGGA TGG-3' and GAPDH-R: 5'-TGCTGTAGCCAAATTCGTTG-3'. The expected fragment sizes according to hg18 are $189 \mathrm{bp}$ and $415 \mathrm{bp}$, respectively. The PCR was performed on a final volume of $25 \mu \mathrm{l}$ using $0.5 \mathrm{U}$ of BioTherm DNA polymerase (GeneCraft), $2 \mathrm{mM}$ of $\mathrm{Mg}$, $50 \mu \mathrm{M}$ of each dNTP, 0.12 $\mu \mathrm{M}$ of each primer and $1 \mu \mathrm{l}$ or $0.5 \mathrm{ul}$ of cDNA (SGK493 and GAPDH, respectively). Finally $10 \mu \mathrm{l}$ of the SGK493 PCR and $5 \mu \mathrm{l}$ of the GAPDH PCR were resolved on a $2 \%$ agarose gel.

The study was conducted in accordance with the Declaration of Helsinki Principles. Ethical approval was obtained for each centre from the appropriate institutional ethics committee and all subjects participating in this study provided informed consent.

\section{Results \\ Pooling GWA}

A genome wide analysis was performed with three pools of DNA from atopic, atopic asthmatics and control individuals. Population characteristics are shown in Table 1. Significant results after correction for 5\% FDR in the GWA of pooled DNA are shown in Table 2 (see Additional file 1: table S1 for the genome-wide surrounding SNPs). Seven SNPs were associated with atopy and one with atopic asthma. From these results, five regions were selected for individual genotyping at a second stage. Three of these regions contained genes putatively related to asthma or atopy: collagen type XXIX alpha 1 (COL29A1, also known as COL6A5, a new member of the collagen family [19]), mitogen-activated protein kinase kinase kinase 5 (MAP3K5, also known as apoptosis signal-regulating kinase 1 (ASK1)), collagen type XVIII alpha 1 (COL18A1) and solute carrier family 19 member 1 (SLC19A1). The other two regions contained genes for 
Table 2: SNPs associated with asthma or atopy with a FDR of $5 \%$ in the pooling-based GWA

\begin{tabular}{cccccccc}
\hline SNP & Chromosome & Position & Comparison & p value & fAco-fAca & Region & Saturation \\
\hline rs2501618 & 1 & 178225432 & Atopy vs. controls & $1.26 \times 10^{-7}$ & 0.26 & CEP350 \\
\hline rs4952590 & 2 & 42130425 & Atopy vs. controls & $4.63 \times 10^{-7}$ & 0.19 & SGK493 (LOC9I46I) & Yes \\
\hline rs7629719 & 3 & 131642624 & Atopy-Asthma vs. controls & $1.11 \times 10^{-7}$ & 0.21 & COL29AI (COL6A5) & Yes \\
\hline rs9292961 & 5 & 20975886 & Atopy vs. controls & $5.38 \times 10^{-7}$ & 0.3 & Segmental Duplications & No \\
\hline rs9376221 & 6 & 137034072 & Atopy vs. controls & $3.94 \times 10^{-7}$ & 0.25 & MAP3K5 (ASKI) & Yes \\
\hline rs7843085 & 8 & 33235086 & Atopy vs. controls & $3.93 \times 10^{-7}$ & 0.26 & NT_007995.50 & Yes \\
\hline rs13273924 & 8 & 33246541 & Atopy vs. controls & $7.56 \times 10^{-7}$ & 0.26 & NT_007995.50 & Yes \\
\hline rs2330183 & 21 & 45777720 & Atopy vs. controls & $1.59 \times 10^{-7}$ & 0.28 & SLCI9AI
\end{tabular}

fAco (allele frequency in controls); fAca (allele frequency in cases); CEP350: centrosomal protein $350 \mathrm{kDa}$; SGK493: protein kinase-like protein SgK493; COL29A I: collagen, type XXIX, alpha I; MAP3K5: mitogen-activated protein kinase kinase kinase 5; ASK I: apoptosis signal-regulating kinase I; SLCI 9AI: solute carrier family 19 (folate transporter), member I

which no obvious functionality could be inferred: Homo sapiens protein kinase-like protein SgK493 (SGK493) and a predicted gene in chromosome 8 (NT_007995.50) which presented two consecutive SNPs associated with atopy in the pooled analysis. Additional SNPs surrounding these six markers were genotyped at an individual level (see Additional file 1: table S2, for the results of the 53 SNPs genotyped at an individual level).

\section{Individual genotyping}

A summary of the positive results on individual samples for atopy is shown in Table 3. SNPs found to be significant for atopy at individual level in both pooling samples and replication set were located in SGK493 and MAP3K5 genes. Variants in COL18A1 and COL29A1 were not consistently replicated in both samples, although a variant in the COL18A1 (at 5' of the SLC19A1 region) was signifi-

Table 3: Summary of the SNPs significantly associated with atopy at an individual level

\begin{tabular}{|c|c|c|c|c|c|c|c|}
\hline \multirow[b]{2}{*}{ SNP } & \multirow[b]{2}{*}{ Gene } & \multirow[b]{2}{*}{ MAF } & \multirow[b]{2}{*}{ Chromosome } & \multirow[b]{2}{*}{ Position (build36) } & \multicolumn{3}{|c|}{ ECHRS Atopy vs. controls } \\
\hline & & & & & Pooling & Replication & Combined \\
\hline rsIII24858* & SGK493 & 0.367 & 2 & 42113828 & 0.0021 & 0.0318 & $0.0007 \dagger$ \\
\hline rs I3409978* & SGK493 & 0.122 & 2 & 42119206 & $0.0007 \dagger$ & 0.0124 & $0.0001 t$ \\
\hline rs4952590*** & SGK493 & 0.141 & 2 & 42130425 & $0.0001 t$ & $0.0008^{t}$ & $1.9 \times 10^{-6 \dagger}$ \\
\hline rs|440095 & SGK493 & 0.379 & 2 & 42143170 & 0.0098 & 0.1078 & 0.0105 \\
\hline rs10934938 & COL29AI & 0.23 & 3 & 131596325 & 0.1158 & 0.0291 & 0.0056 \\
\hline rs9402839* & MAP3K5 & 0.138 & 6 & 137036903 & 0.0355 & 0.0524 & 0.0099 \\
\hline rs9494554** & MAP3K5 & 0.095 & 6 & 137038675 & 0.0306 & 0.0035 & $0.0005 t$ \\
\hline rs $12483377^{*}$ & COLIBAI & 0.108 & 21 & 45755537 & 0.0046 & 0.0607 & 0.0030 \\
\hline
\end{tabular}

*SNPs found associated in the pooling-based GWA or their perfect tags $\left(r^{2}=0.8\right)$ used as substitutes in the genotyping design.

**SNPs that remained significant after a 5\% FDR in the pooling-based GWA

tP-values that remain significant after Bonferroni correction (46 SNPs, $p \leq 0.00 I$ ) 
cant in pooling samples ( $\mathrm{p}=0.005)$ and border significant in the replication $(\mathrm{p}=0.06)$. For variants listed in Table 3, the combined analysis of samples from pooling and the additional case control set, led to lower p values, indicating that genetic effects are in the same direction in both sets [20].

The region in chromosome 2 containing the SGK493 gene showed the SNPs with the most significant $\mathrm{p}$ values in the association with atopy, and similar results were observed for the subset of atopic individuals with asthma (see Additional file 1: table S2). The variant rs4952590 remain significant even after Bonferroni correction (46 SNPs, $\mathrm{p} \leq$ 0.001 ) in the both pooling and replication samples. This variant was associated with a reduction in risk of atopy in both samples (odds ratio $(\mathrm{OR})=0.18,95 \%$ confidence interval (CI) 0.07-0.47 for pooling sample and $\mathrm{OR}=0.52$, $95 \%$ CI $0.35-0.79$ for replication sample). In addition, variant rs13409978 in the pooling sample and three SNPs (rs11124858, rs13409978, rs4952590) remained significant in the combined analysis after Bonferroni correction. Figure 1 shows the representation of the linkage disequilibrium (LD) pattern for the 6 SNPs of SGK493 genotyped for all individuals of the sample $(n=699)$. Two blocks of disequilibrium were defined according to Gabriel et al. [18] method implemented in Haploview. Complete LD pattern of the region derived from HapMap data is summarized in the Additional file 1: figure S1. Haplotype analysis using the six SNPs (Table 4) confirms the results obtained in the single marker analysis. The only haplotype containing $\mathrm{C}$ allele of rs13409978 and $\mathrm{T}$ allele of rs4952590 is the only significantly associated with atopy $(\mathrm{p}=0.00005)$. These SNPs are in high LD $\left(\mathrm{D}^{\prime}=0.97\right.$ and $\left.\mathrm{r}^{2}=0.88\right)$. The expression pattern of SGK493 presented in Figure 2 shows that SGK493 is ubiquitously expressed in all the human tissues analyzed.

The intronic SNP rs9494554, located within MAP3K5, was associated with atopy in the pooling and replication samples, and remained significant in the combined analysis after Bonferroni correction. A similar association for rs9494554 was observed in the subsample of atopic asth- matic individuals (see Additional file 1: table S2). Other SNP in MAP3K5 (rs9402839) was nominally significant for atopy in pooling sample and border significant in the replication step.

\section{Discussion}

We performed a two-stage DNA pooling-based GWA for asthma and atopy, evaluating a genome-wide panel of markers in a subset of subjects using a DNA pooling strategy, and in a second stage we evaluated the most promising markers at an individual level in the individual pooled samples as well as in a second set of cases and controls. We identified SGK493 as a new potential gene associated to atopy. MAP3K5, COL18A1 and COL29A1 genes were found to lesser extent associated with atopy.

The most significant results were observed for SGK493 gene, in chromosome 2 , with similar results for atopy and for atopic asthma, probably due to sharing predisposing factors [1]. Significant polymorphisms were located in two different linkage disequilibrium blocks. The first block includes the putative promoter region of SGK493 (5' upstream) and the second block covers part of the gene and the 3' untranslated region (UTR) and 3'downstream region (Figure 1). Haplotype based analysis confirmed results obtained in single marker analysis. We could not elucidate which is the functionality of these SNPs and a fine mapping of the region within a more powered sample would be required. We cannot exclude that the functional variant could be tagged by these SNPs and located in another close gene. SGK493 gene was identified during the creation of a catalogue of human protein kinases [21], but its particular function is unknown. SGK493 could be involved in pathological states as protein kinases mediate most of the signal transduction in eukaryotic cells [22]. We reported that SGK493 gene is ubiquitously expressed in human tissues, however a higher expression on lung and uterus was previously identified by microarray experiment from 73 human tissues http://biogps.gnf.org/[23]. Recently, the knock out of Sgk493 (also known as Pkdcc) in mouse has been described presenting extreme phenotypes that are not a priori related with atopy or asthma.

Table 4: Haplotype analysis and multiple regression for SGK493 gene variants.

\begin{tabular}{llllllll}
\hline rsIII24858 & rsI3409978 & rsl7029I2I & rs4952590 & rs2424 & rsI440095 & Frequency & p-value \\
\hline A & A & A & C & A & T & 0.36 & reference \\
\hline$*$ & $*$ & G & $*$ & $*$ & $*$ & 0.22 & 0.128 \\
\hline $\mathrm{G}$ & $*$ & $*$ & $*$ & T & C & 0.17 & 0.506 \\
\hline $\mathrm{G}$ & C & $*$ & T & $*$ & C & 0.12 & 0.00005 \\
\hline
\end{tabular}

* Same allele to reference haplotype (most frequent) 


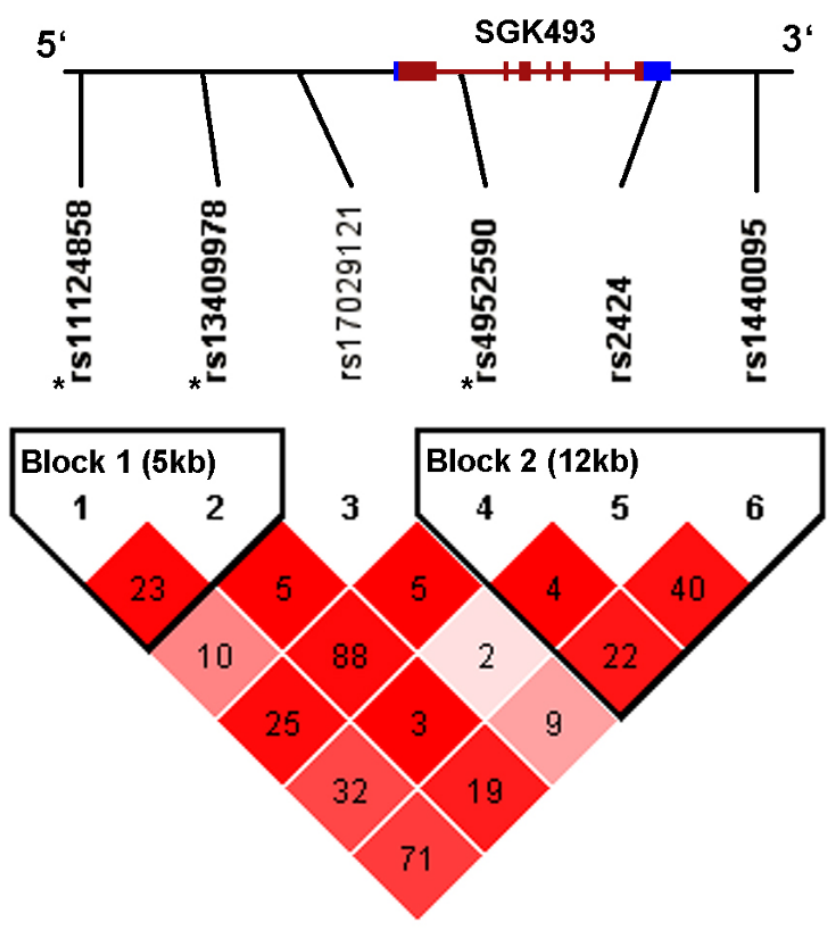

Figure I

Linkage disequilibrium pattern and haplotype blocks of SNPs in the SGK493 gene using genotyping data in this study. Colors correspond to $\mathrm{D}^{\prime}$ and numbers in each cell to $r^{2}$ parameter. Blocks were defined with method described by Gabriel et al. [18]. In red is marked the coding region of the SGK493 gene (lines are introns and boxes exons) and blue boxes show the untranslated (UTR) regions of the gene. * SNPs found associated in the individual pooled samples and replication sample (Table 3).

The knock out mice showed abnormal respiration and died within a day possibly due to cleft palate [24].

Another kinase MAP3K5 was also related to atopy as well as atopic asthma in this study. MAP3K5 encodes for a member of the mitogen-activated protein kinase family that regulates the activation of the transcription factor activator protein-1 (AP1) in leukotriene D (4) (LTD(4)) stimulated airway smooth muscle cells and in nitric oxygen (NO) stimulated bronchial epithelial cells $[25,26]$. AP1 play a role in the production of airway inflammation [26].

Polymorphisms in COL18A1 and COL29A1 were not consistently replicated. The COL18A1 polymorphism shown to be significantly associated with atopy in the pooling sample is located at the 3 ' coding region of the gene. COL18A1 encodes a protein expressed in epithelial and endothelial basement membranes, involved in regulation of angiogenesis and endothelial cell proliferation [27-29].
The COL29A1 polymorphism was previously found to be associated with atopic dermatitis [15]. Finally, a region situated in chromosome 8 , which contains a predicted gene (NT_007995.50), was also analyzed in detail. Nominally associations were observed in the pooling sample for atopy, but they were not replicated.

Signals described in previous GWA for atopy, asthma and related phenotypes have not been detected in this study [3-5]. The lack of replication of the GWA results can be caused by differences in the definition used to classify affected individuals, genetic coverage of the genome and the $\mathrm{p}$ value threshold [30]. In particular, the region detected by Moffatt et al[3] on chromosome 17 has been associated with childhood onset asthma, while in this study the asthmatic individuals were selected independently of their asthma onset. We have not detected the FCER1A region identified by Weidinger et al[5] for atopy, but a different genotyping platform was used.

Despite significant replication values of most promising SNPs found in the GWA in pooled DNA, we acknowledge some limitations as the lack of replication for some of the loci identified in the pooling based analysis. Non-replication of the initial findings is a common feature of the initial findings in GWA studies [31], mainly due to heterogeneity in the aetiology of the disease and biases. In addition, the impossibility of detection and adjustment by potential confounders in the analysis of DNA pooling could produce the inconsistencies observed.

Another of the main limitation is the sample size for pool construction and replication. Regarding DNA pooling, the method for measure allele frequency differences corrects by the number of subjects included in each pool [11]. After this correction we obtained some signals at very low p-values, which reinforce the strength of these results. Given limited sample, our study was able to detect variants with larger effects in this population and probably other variants with smaller effects would not be detected. False positives are controlled in the replication phase by individual analysis. Other pooling strategies such as use of different sub-samples of pools would allow capture more biological variation. We acknowledge that with having larger pools we may be losing positive signals but we consider this as a less crucial issue for this analysis. Power calculation shows that replication sample was powered to detect reported associations given the parameters observed in the analysis of individual pooling data (see Additional file 1: table S3). For this reason, some of the results were replicated and significance for a SNP in SGK493 reaches Bonferroni level. Although this type of correction by multiple testing is over-conservative and induces false negatives, it is a good indicator of the significance level. In addition to the significance level, some of 


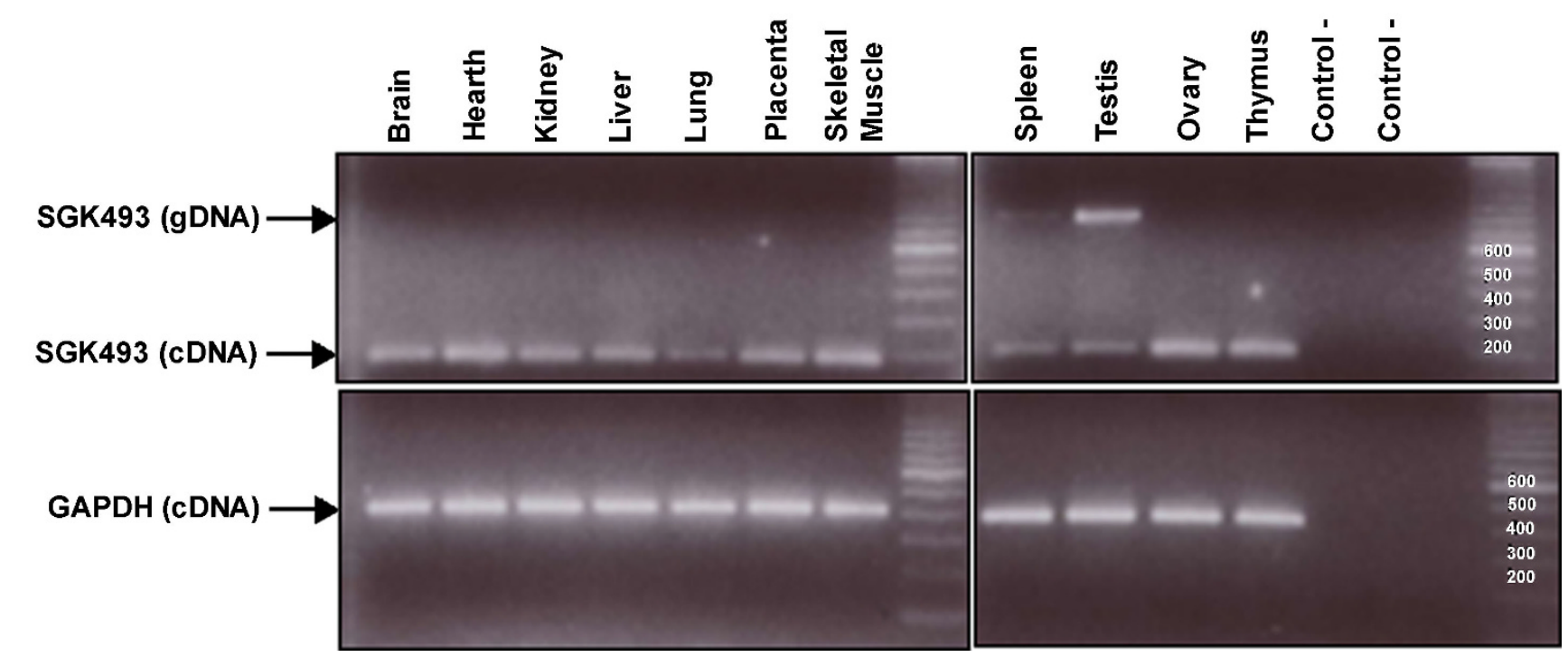

Figure 2

SGK493 expression in a panel of human tissues. Primers are situated in exon 3 and 4 according to the RefSeq Genes. All the human tissues tested express SGK493. The amplification of GAPDH has been used as a control of the cDNA quality. In spleen and testis a second fragment of approximately $800 \mathrm{bp}$ is observed. This fragment, based on its size, could be the amplification of SGK493 from genomic DNA.

the results were replicated in the additional sample and replication is considered essential to establish the validity of associations [32].

Finally, another limitation is the small number of regions analyzed in detail in the individual analysis. We discarded complex regions with segmental duplications and putative insertions or deletions [13] because their complexity could a priori result in reducing power to detect associations due to non-classical inheritance patterns of markers located on them. In contrast, disease definition is a positive feature of this study since it is highly homogeneous among patients derived from different ECHRS centre, and this is a keystone for valid inferences in association studies.

\section{Conclusion}

In summary, we identified a region related to atopy on chromosome 2p21 that contains SGK493 gene. A nonsynonymous SNP in COL18A1, intronic variants in MAP3K5 and a polymorphism in COL29A1, previously related to atopic dermatitis, were also associated with atopy, but not consistently replicated. Follow-up analysis in larger sample sets, as well fine mapping will be needed to validate these susceptibility loci.

\section{Abbreviations}

SGK493: protein kinase-like protein SgK493; COL29A1: collagen, type XXIX, alpha 1; MAP3K5: mitogen-activated protein kinase kinase kinase 5; ASK1: apoptosis signalregulating kinase 1; SLC19A1: solute carrier family 19 (folate transporter), member 1; COL18A1: collagen, type XVIII, alpha 1; GWA: Genome-Wide Analysis; ECRHS: European Community Respiratory Health Survey; IgE: Immunoglobulin E; SNP: single nucleotide polymorphisms; FDR: false discovery rate; HWE: Hardy-Weinberg equilibrium; LD: Linkage disequilibrium; LTD(4): transcription factor activator protein-1 (AP1) in leukotriene D (4); NO: nitric oxygen; kDa: kilodaltons; VEGF: vascular endothelial growth factor; FCER1A: Fc IgE receptor, alpha polypeptide.

\section{Competing interests}

The authors declare that they have no competing interests.

\section{Authors' contributions}

FCG participated in the design of the study, prepared the DNA for pooling, performed the statistical analysis and wrote the first draft of the manuscript; $\mathrm{MB}$ participated in the design of the second stage of the study, prepared the DNA, performed the genotyping of the individual samples and wrote the first draft of the manuscript; JRG participated in the design of the second stage of the study and assisted with the statistical analysis; MK conceived the study, participated in the design of the study and participated in writing the manuscript; DJ was responsible for design and coordination of the ECRHS study in UK and participated in writing the manuscript; JH was responsible 
for design and coordination of the ECRHS study in Germany and participated in writing the manuscript; JMA was responsible for design and coordination of the ECRHS study in Spain and participated in writing the manuscript; MW was responsible for DNA biobank, participated in the design of the study and participated in writing the manuscript; XE participated in the design of the study and participated in writing the manuscript; $\mathrm{RdC}$ conceived the study, coordinate the study and participated in writing the manuscript; All authors read and approved the final manuscript.

\section{Additional material}

\section{Additional file 1}

Additional file is in acrobat reader file format. Contains 3 tables and 1 Figure: $\bullet$ Additional table S1 - SNPs associated with asthma or atopy with a FDR of 5\% and the surrounding region in the pooling-based GWA. $\bullet$ Additional table S2 - Results of the association between the 53 SNPs and asthma or atopy at an individual level. - Additional table S3 - Statistical power calculation for replication using Quanto software v1.2.4 http:// hydra.usc.edu/gxe for significance (two-sided) of 0.05 and additive genetic model. Additional figure S1 - Linkage disequilibrium pattern for the HapMap CEPH population of the region flanking SGK493 gene (10 $k b$ at $5^{\prime}$ and $\left.3^{\prime}\right)$ based on the method of Gabriel et al [18] implemented in Haploview.

Click here for file

[http://www.biomedcentral.com/content/supplementary/14712350-10-128-S1.PDF]

\section{Acknowledgements}

Part of this work was supported by the Spanish Ministry for Science and Innovation [MTM2008-02457 to JRG and FC; and SAF2008-00357 to XE, $M B$ and $R$ de $C$ ], and Public Health and Epidemiology Network Biomedical Research Center (CIBERESP). The genotyping and part of the DNA extractions were performed at the Spanish National Genotyping Centre (CEGEN).

\section{References}

I. Ober C, Hoffjan S: Asthma genetics 2006: the long and winding road to gene discovery. Genes Immun 2006, 7:95-100.

2. Denham S, Koppelman GH, Blakey J, Wjst M, Ferreira MA, Hall IP, et al:: Meta-analysis of genome-wide linkage studies of asthma and related traits. Respir Res 2008, 9:38.

3. Moffatt MF, Kabesch M, Liang L, Dixon AL, Strachan D, Heath S, et al:: Genetic variants regulating ORMDL3 expression contribute to the risk of childhood asthma. Nature 2007, 448:470-473.

4. Wilk JB, Walter RE, Laramie JM, Gottlieb DJ, O'Connor GT: Framingham Heart Study genome-wide association: results for pulmonary function measures. BMC Med Genet 2007, 8(Suppl I):S8.

5. Weidinger S, Gieger C, Rodriguez E, Baurecht H, Mempel M, Klopp $N$, et al.: Genome-wide scan on total serum IgE levels identifies FCERIA as novel susceptibility locus. PLoS Genet 2008, 4:e1000166.

6. Pearson JV, Huentelman MJ, Halperin RF, Tembe WD, Melquist S, Homer N, et al:: Identification of the genetic basis for complex disorders by use of pooling-based genomewide single-nucleotide-polymorphism association studies. Am J Hum Genet 2007, 80:126-139.
7. Steer S, Abkevich V, Gutin A, Cordell HJ, Gendall KL, Merriman ME, et al.: Genomic DNA pooling for whole-genome association scans in complex disease: empirical demonstration of efficacy in rheumatoid arthritis. Genes Immun 2007, 8:57-68.

8. Zhao Y, Wang S: Optimal DNA pooling-based two-stage designs in case-control association studies. Hum Hered 2009, 67:46-56.

9. Burney PG, Luczynska C, Chinn S, Jarvis D: The European Community Respiratory Health Survey. Eur Respir J 1994, 7:954-960.

10. Craig DW, Huentelman MJ, Hu-Lince D, Zismann VL, Kruer MC, Lee $A M$, et al:: Identification of disease causing loci using an arraybased genotyping approach on pooled DNA. BMC Genomics 2005, 6:138.

II. Visscher PM, Le Hellard S: Simple method to analyze SNPbased association studies using DNA pools. Genet Epidemiol 2003, 24:29I-296.

12. Benjamini Y, Drai D, Elmer G, Kafkafi N, Golani I: Controlling the false discovery rate in behavior genetics research. Behav Brain Res 200I, I 25:279-284

13. Kidd JM, Cooper GM, Donahue WF, Hayden HS, Sampas N, Graves $\mathrm{T}$, et al.: Mapping and sequencing of structural variation from eight human genomes. Nature 2008, 453:56-64.

14. Barrett JC, Fry B, Maller J, Daly MJ: Haploview: analysis and visualization of LD and haplotype maps. Bioinformatics 2005, 21:263-265.

15. Soderhall C, Marenholz I, Kerscher T, Ruschendorf F, Esparza-Gordillo J, Worm M, et al: Variants in a novel epidermal collagen gene (COL29AI) are associated with atopic dermatitis. PLOS Biol 2007, 5:e242.

16. R Development Core Team: R: A language and environment for statistical computing. R Foundation for Statistical Computing. Vienna, Austria 2007 [http://www.R-project.org]

17. Heath SC, Gut IG, Brennan P, McKay JD, Bencko V, Fabianova E, et al.: Investigation of the fine structure of European populations with applications to disease association studies. Eur $\mathrm{J}$ Hum Genet 2008, 16:1413-1429.

18. Gabriel SB, Schaffner SF, Nguyen H, Moore JM, Roy J, Blumenstiel B, et al:: The structure of haplotype blocks in the human genome. Science 2002, 296:2225-2229.

19. Gara SK, Grumati P, Urciuolo A, Bonaldo P, Kobbe B, Koch M, et al.: Three novel collagen VI chains with high homology to the alpha3 chain. J Biol Chem 2008, 283:10658-10670.

20. Skol AD, Scott LJ, Abecasis GR, Boehnke M: Joint analysis is more efficient than replication-based analysis for two-stage genome-wide association studies. Nat Genet 2006, 38:209-213.

21. Manning G, Whyte DB, Martinez R, Hunter T, Sudarsanam S: The protein kinase complement of the human genome. Science 2002, 298:1912-1934.

22. Cohen P: Protein kinases--the major drug targets of the twenty-first century? Nat Rev Drug Discov 2002, I:309-3I5

23. Su Al, Wiltshire T, Batalov S, Lapp H, Ching KA, Block D, et al:: A gene atlas of the mouse and human protein-encoding transcriptomes. Proc Natl Acad Sci USA 2004, 10 1:6062-6067.

24. Imuta Y, Nishioka N, Kiyonari H, Sasaki H: Short limbs, cleft palate, and delayed formation of flat proliferative chondrocytes in mice with targeted disruption of a putative protein kinase gene, Pkdcc (AW548 I 24). Dev Dyn 2009, 238:2 10-222.

25. Kumasawa F, Hashimoto S, Onose A, Jibiki I, Mizumura K, Matsumoto $\mathrm{K}$, et al.: Apoptosis signal-regulating kinase $\mathbf{I}$ in leukotriene $D(4)$-induced activator protein- $I$ activation in airway smooth muscle cells. Eur J Pharmacol 2005, 5 1 7: I I-16.

26. Jibiki I, Hashimoto S, Maruoka S, Gon Y, Matsuzawa A, Nishitoh H, et al.: Apoptosis signal-regulating kinase I-mediated signaling pathway regulates nitric oxide-induced activator protein- $I$ activation in human bronchial epithelial cells. Am J Respir Crit Care Med 2003, 167:856-861.

27. Marneros AG, Olsen BR: Physiological role of collagen XVIII and endostatin. FASEB J 2005, 19:716-728.

28. Asai K, Kanazawa H, Otani K, Shiraishi S, Hirata K, Yoshikawa J: Imbalance between vascular endothelial growth factor and endostatin levels in induced sputum from asthmatic subjects. J Allergy Clin Immunol 2002, I I 0:57I I-575.

29. Suzaki Y, Hamada K, Sho M, Ito T, Miyamoto K, Akashi S, et al.: A potent antiangiogenic factor, endostatin prevents the devel- 
opment of asthma in a murine model. J Allergy Clin Immunol 2005, I 16: I 220-1227.

30. loannidis JP, Patsopoulos NA, Evangelou E: Heterogeneity in meta-analyses of genome-wide association investigations. PLOS ONE 2007, 2:e84I.

31. loannidis JP: Non-replication and inconsistency in the genomewide association setting. Hum Hered 2007, 64:203-2I3.

32. Chanock SJ, Manolio T, Boehnke M, Boerwinkle E, Hunter DJ, Thomas G, et al:: Replicating genotype-phenotype associations. Nature 2007, 447:655-660.

\section{Pre-publication history}

The pre-publication history for this paper can be accessed here:

http://www.biomedcentral.com/1471-2350/10/128/pre pub

Publish with Bio Med Central and every scientist can read your work free of charge

"BioMed Central will be the most significant development for disseminating the results of biomedical research in our lifetime. "

Sir Paul Nurse, Cancer Research UK

Your research papers will be:

- available free of charge to the entire biomedical community

- peer reviewed and published immediately upon acceptance

- cited in PubMed and archived on PubMed Central

- yours - you keep the copyright

Submit your manuscript here:

http://www.biomedcentral.com/info/publishing_adv.asp 